Tropical Journal of Pharmaceutical Research January 2021; 20 (1): 75-81

ISSN: $1596-5996$ (print); 1596-9827 (electronic)

(C) Pharmacotherapy Group, Faculty of Pharmacy, University of Benin, Benin City, 300001 Nigeria.

Original Research Article

http://dx.doi.org/10.4314/tjpr.v20i1.12

\title{
Swertiamarin exerts anticancer effects on human cervical cancer cells via induction of apoptosis, inhibition of cell migration and targeting of MEK-ERK pathway
}

\author{
Xinxiang Wang ${ }^{1}$, Tao Wang ${ }^{2 *}$ \\ ${ }^{1}$ Reproductive Medicine Centre, Baoji Maternal and Child Health Care Hospital, Baoji, 721000, ${ }^{2}$ Center For Assistant \\ Reproductive Technology, Northwest Women's and Children's Hospital, Xi'an, Shaanxi 710061, China \\ *For correspondence: Email: wangtao29@126.com; Tel/Fax: 0086-029-87219120
}

Sent for review: 18 November 2019

Revised accepted: 12 December 2020

\begin{abstract}
Purpose: To investigate the anticancer effects of swertiamarin against taxol-resistant human cervical cancer cells.

Method: Cell viability was investigated using 3-(4,5-dimethylthiazol-2-yl)-2,5-diphenyl tetrazolium bromide (MTT) assay while colony survival was evaluated by clonogenic assay. Apoptotic cell death was assessed by AO/ETBR staining and western blotting techniques. The levels of reactive oxygen species (ROS) were measured using 2,7, dicholoro dihydrofluoresceindiacetate (H2DCFDA) staining. Cell migration and invasion were monitored with Transwell chamber assay. Western blotting assay was used to determine the expression levels of proteins of the MEK/ERK signaling pathway.

Results: Swertiamarin induced dose- and time-dependent inhibition of proliferation of HeLa cervical cancer cells $(p<0.05)$. It also suppressed the colony formation potential of HeLa cells, and induced various structural modifications in HeLa cells. Swertiamarin exposure resulted in the formation of earlyapoptotic, late-apoptotic and necrotic cells, and significant modulation of apoptosis-allied proteins. It was observed that the migration and invasion of HeLa cells were potentially suppressed in dose-reliant fashion by swertiamarin. Western blotting results showed that the expressions of $p$-MEK and $p$-ERK were markedly reduced, while those of MEK and ERK were unaffected $(p<0.05)$.

Conclusion: Swertiamarin exerts in vitro anticancer activity against cervical cancer cells (HeLa). Thus, it is promising for use in cervical cancer chemotherapy. However, there is need for confirmation of these findings through further in vivo and in vitro investigations.
\end{abstract}

Keywords: Swertiamarin, Gentianaceae, Triterpene Sapogenin, Cervical cancer

This is an Open Access article that uses a fund-ing model which does not charge readers or their institutions for access and distributed under the terms of the Creative Commons Attribution License (http://creativecommons.org/licenses/by/4.0) and the Budapest Open Access Initiative (http://www.budapestopenaccessinitiative.org/read), which permit unrestricted use, distribution, and reproduction in any medium, provided the original work is properly credited.

Tropical Journal of Pharmaceutical Research is indexed by Science Citation Index (SciSearch), Scopus, International Pharmaceutical Abstract, Chemical Abstracts, Embase, Index Copernicus, EBSCO, African Index Medicus, JournalSeek, Journal Citation Reports/Science Edition, Directory of Open Access Journals (DOAJ), African Journal Online, Bioline International, Open-J-Gate and Pharmacy Abstracts

\section{INTRODUCTION}

Natural products possess great medicinal potential and enormous biological activities. Natural products contain a rich variety of chemical entities with huge structural diversity that are beneficial in addressing different health issues [1-4]. Enicostemma littorale Blume is a naturally prevalent herb belonging to the family of Gentianaceae, often termed Nagajihva in Ayurveda [5]. Several medicinal benefits of Enicostemma littorale Blume have been 
reported. These include anticancer, antiinflammatory and hepatoprotective properties [68]. The plant contains a variety of biochemical compounds, amongst which is swertiamarin triterpene [9-11]. Swertiamarin is a bioactive compound which has been reported to exert antidiabetic effects, as well as regulatory effects on adipose and hepatic tissue gene expressions $[12,13]$.

Cervical cancer (CC) is a deadly tumor of the female reproductive system. It has been ranked as the $4^{\text {th }}$ most frequent cancer and the $4^{\text {th }}$ leading cause of cancer-related death among women [14]. Statistics revealed that there were 0.5 million new cases of $\mathrm{CC}$ and 0.25 million deaths caused by CC in 2012. This accounted for $8 \%$ of the entire cancer-associated deaths in 2012 [15]. Nearly $90 \%$ of CC-related mortality and $70 \%$ of CC cases are reported in developing countries [16]. It has been reported that countries which are economically weak have higher cases of cancer-related deaths [17]. The extensive use of CC screening programs has led to marked reduction in the incidence of the disease [18]. The major risk factors for $\mathrm{CC}$ are prolonged HPV infection, HIV infection and smoking [19]. However, although several other factors have been implicated, the detailed pathogenesis of CC has not been completely understood till date [20].

Several different treatment strategies are applied worldwide for management of CC. It has been reported that $\mathrm{CC}$ responds positively to radiation therapy. Thus, radiation therapy is globally used extensively in treatment of almost every type of CC. Other treatment options include chemotherapy, targeted therapy and surgical resection. However, notwithstanding these available treatment strategies, CC management still remains a big challenge to researchers and scientists.

The overall survival of CC cases is very poor. Therefore, the search for promising chemotherapeutic agents that may produce better effects and lower side-effects, is on-going. In the present study, the anticancer effect of swertiamarin on human taxol-resistant CC was investigated with respect to ROS generation, induction of mitochondrial-dependent apoptosis, inhibition of cell migration and invasion, and targeting MEK-ERK signaling pathway.

\section{EXPERIMENTAL}

\section{Determination of cytotoxicity of swertiamarin}

The effect of swertiamarin on the proliferation of CC HeLa cells was determined using 3-(4,5- dimethylthiazol-2-yl) -2,5-diphenyl tetrazolium bromide (MTT) assay. HeLa cells were seeded in 96-well plate at a density of $1 \times 10^{4}$ cells/well in $200 \mu \mathrm{L}$ of complete culture medium. The cells were thereafter treated for $48 \mathrm{~h}$ and $72 \mathrm{~h}$ with gradient doses of swertiamarin, i.e., 10, 20, 40 and $80 \mu \mathrm{M}$. After incubation at $37{ }^{\circ} \mathrm{C}$ in a humidified incubator, $20 \mu \mathrm{L}$ of stock solution of MTT was added to each well. After further incubation, the medium was carefully removed, and the cells were shaken with DMSO $(150 \mu \mathrm{L})$ for $15 \mathrm{~min}$ to dissolve the formazan crystals formed. Finally, the absorbance of each well was read at 490 nm in an ELISA microplate reader. Untreated HeLa cells (control) were taken as $100 \%$ viable.

\section{Clonogenic assay}

The HeLa CC cells were treated with different concentrations of swertiamarin $(10,40$ and 80 $\mu \mathrm{M})$ for $24 \mathrm{~h}$. Thereafter, the colonies were diluted in agar solution $(0.3 \%)$ and reloaded on $35-\mathrm{mm}$ culture plates. Each plate contained about 320 HeLa cell colonies. When the agar solution solidified, cell colonies were left untouched for two weeks. Finally, the cell colonies were enumerated in 2-mm grid culture dish (Corning).

\section{Assessment of morphology of HeLa cells}

Cellular morphology of HeLa CC cells was examined using phase contrast microscopy. HeLa cells were incubated in $60-\mathrm{mm}$ culture dishes at a density of $5 \times 10^{4}$ cells/dish for $48 \mathrm{~h}$. Thereafter, different concentrations of swertiamarin were added to the dishes viz 10, 40 and $80 \mu \mathrm{M}$, and left untouched for $24 \mathrm{~h}$. The swertiamarin treatment was followed by washing with PBS and discarding of culture medium. Finally, changes induced by swertiamarin in HeLa cells were observed and photographed under a phase contrast inverted microscope (Leica DMI 3000B, Germany).

\section{AO/EB-staining for apoptosis analysis}

HeLa cells were harvested in 96-well plate at $75 \%$ confluence. The cells were then subjected to swertiamarin treatment doses of 10,40 and 80 $\mu \mathrm{M}$ for about $24 \mathrm{~h}$. Drug treatment was followed by trypsinization $(20 \mu \mathrm{l})$. The trypsinized cells were sloughed off and transferred to glass slides. Each suspension was stained with $1 \mu \mathrm{L}$ of dual fluorescent staining solution $[100 \mu \mathrm{g} / \mathrm{mL} A O$ and $100 \mu \mathrm{g} / \mathrm{ml}$ EB (Sigma, St. Louis, MO)] and protected with coverslips. Finally, fluorescent microscope (OLYMPUS, Japan) was used to 
visualise the intra- and extracellular morphologies of HeLa cells.

\section{Determination of levels of reactive oxygen species (ROS)}

The $\mathrm{H}_{2}$ DCFDA staining assay was used to determine ROS levels in HeLa CC cells. The cells were cultured and harvested at $80 \%$ confluence, followed by swertiamarin exposure at graded doses viz 10, 40 and $80 \mu \mathrm{M}$. The treated cells were then stained for 30 min with $2 \mu \mathrm{M}$ $\mathrm{H}_{2}$ DCFDA. Finally, the cells were washed using phosphate-buffered saline (PBS), and fluorescence was recorded using a flow cytometer (ACEA NoVoCyte).

\section{Transwell migration and cell invasion assays}

Transwell migration assay was used to determine the migration potential of HeLa cells. In the upper chamber, nearly $3 \times 10^{3}$ HeLa cells were cultured in RPMI-1640 medium (600 $\mu \mathrm{L})$. The lower chambers contained medium and FBS (10\%) only. The upper chamber was treated with different doses of swertiamarin (10, 40 and 80 $\mu \mathrm{M})$ for $24 \mathrm{~h}$. The Transwell chambers were then incubated for $6 \mathrm{~h}$ at $37^{\circ} \mathrm{C}$. The upper chambers were thereafter cleaned with a cotton swab to remove non-migrated cells. The migrated cells were fixed and stained with $4 \%$ formalin and 0.1 $\%$ crystal violet for $20 \mathrm{~min}$. Different fields were photographed using microscopy at x200. A similar procedure was performed for cell invasion assay, except that Transwell chambers were coated with Matrigel.

\section{Western blotting assay}

Protein expressions in HeLa CC cells were assayed with western blotting. The cells were treated with graded doses of swertiamarin viz 10 , 40 and $80 \mu \mathrm{M}$., and thereafter lysed with lysis buffer. The protein content of the lysate was measured using BCA method. Then, $10-40 \mu \mathrm{g}$ of protein samples were separated on SDS-PAGE and loaded to PVDF membranes. The membranes were treated with primary antibodies against BAX, BCL-2, Cyt-c, caspase-3, caspase9, MEK, and ERK overnight at $4^{\circ} \mathrm{C}$. This was followed by incubation of the membrane with HRP-linked secondary antibody at room temperature for $1 \mathrm{~h}$. Finally, signals were detected using chemiluminescence reagent kit (Amersham Pharmacia, Piscataway, NJ).

\section{Statistical analysis}

Experimental data are presented as mean $\pm S D$. Student's $t$-test was used for determination of statistical significance, while comparison among multiple groups was performed with ANOVA. Values of $p<0.05$ were considered reflective of statistically significant differences.

\section{RESULTS}

\section{Swertiamarin exerted anti-cell proliferative effects on HeLa cells}

The proliferation of HeLa CC cells after swertiamarin treatment was determined using MTT assay. The results revealed that swertiamarin inhibited proliferation in a concentration- and time-dependent manner (Figure 1). Cell proliferation was reduced to nearly $20 \%$ after $48 \mathrm{~h}$ exposure to swertiamarin, relative to control. It was also observed that cell proliferation was reduced to nearly $5 \%$ by higher swertiamarin doses after $72 \mathrm{~h}$ of exposure (Figure 2).

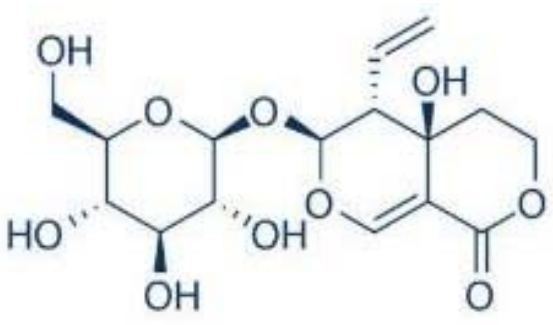

Figure 1: Chemical structure of swertiamarin, a triterpene sapogenin

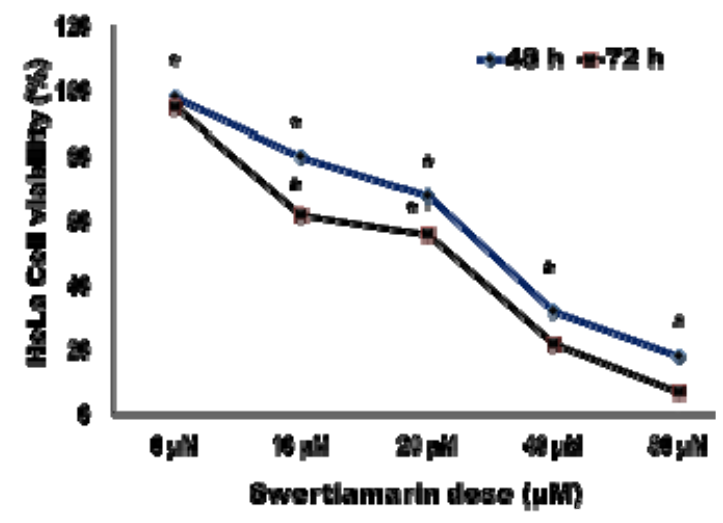

Figure 2: Effect of swertiamarin on the viability of HeLa cervical cancer cells. Data are shown as mean \pm $\mathrm{SD}(\mathrm{n}=3) ; p<0.05$

\section{Swertiamarin inhibited cell colony formation of HeLa cells}

Since swertiamarin induced anti-cell growth effects on HeLa cells, its effect on cell colony formation was determined using clonogenic assay. The results revealed dose-dependent 
suppressive effects of swertiamarin on HeLa cell colonies (Figure $3 \mathrm{~A}$ ). The numbers of cell colonies observed at swertiamarin doses of 75 at 10, 40 and $80 \mu \mathrm{M}$, were 325, 270 and 200, respectively (Figure $3 \quad \mathrm{~B})$. Therefore, swertiamarin induced potent and concentrationdependent inhibition of HeLa cell colony formation.

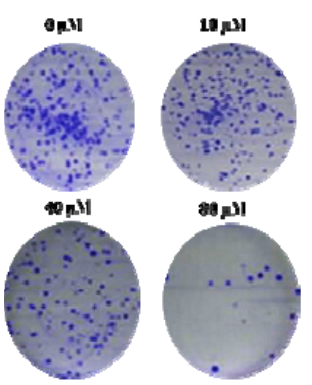

A

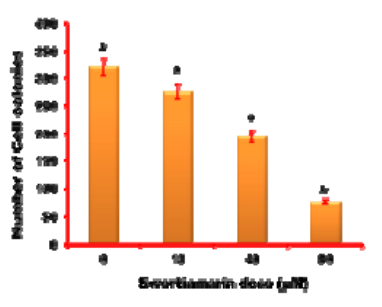

B.

Figure 3: (A) Images of culture dishes showing HeLa cell colonies after performing clonogenic assay at indicated doses. (B) Graphical representation of the effect of swertiamarin on cell colony formation

\section{Swertiamarin induced structural changes in HeLa cells}

The effect of swertiamarin treatment on HeLa cell structure was studied via phase contrast microscopy. The results indicated that swertiamarin induced dose-dependent morphological modifications in HeLa cells, as depicted by presence of condensed nuclei, membrane rupture, membrane blebbing and cell disintegration (Figure 4). These structural modifications indicated apoptotic cell death.

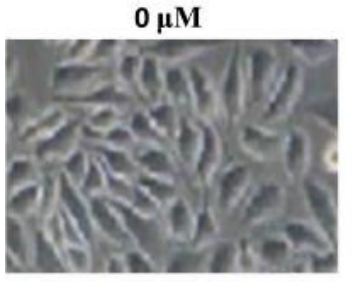

$40 \mu \mathrm{M}$
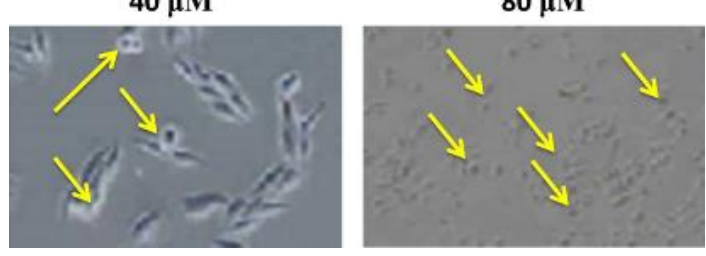

Figure 4: Photomicrographs captured under phase contrast inverted microscope, showing changes in cellular morphology of HeLa cells after swertiamarin exposure. The experiments were performed in triplicate

\section{Swertiamarin induced apoptosis of HeLa cells}

Structural modifications indicative of apoptotic cell death were observed in HeLa cells under phase contrast microscopy. Therefore, AO/ETBR staining assay was performed to analyse swertiamarin-induced apoptotic cell death. The results revealed necrotic, as well as late and early apoptotic cells with orange-red, orange, and yellow-green fluorescence, respectively (Figure 5). The number of necrotic cells was significantly increased by increases in drug doses $(10,40$ and $80 \mu \mathrm{M})$. Thus, AO/EB staining revealed that the anti-proliferative effects and structural modifications were apoptosismediated. Furthermore, to verify if the cell death in HeLa cells was apoptosis-related, western blotting was used to measure the expressions of apoptosis-related proteins. The results revealed that swertiamarin downregulated the expressions of $\mathrm{Bcl}-2$ and significantly upregulated the expressions of Bax, Cyt-c, caspase-3 and caspase-9 (Figure 6). Thus, the results from western blotting assay were consistent with the earlier results showing that swertiamarin induced mitochondrion-mediated apoptotic cell death.

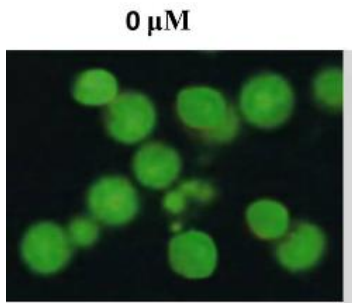

$40 \mu \mathrm{M}$

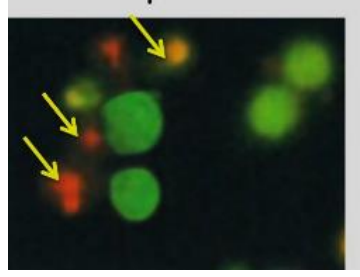

$10 \mu \mathrm{M}$

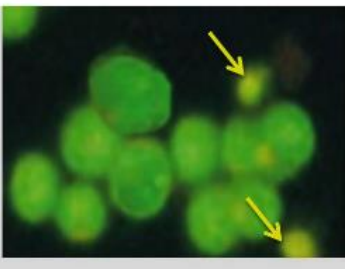

$80 \mu \mathrm{M}$

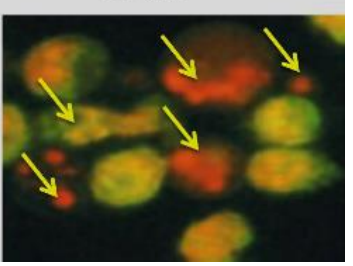

Figure 5: Results of AO/ETBR staining of HeLa cells treated with swertiamarin at indicated doses. The apoptotic cells are indicated with arrows. Early apoptotic cells produced yellow fluorescence, whereas late apoptotic cells produced orange fluorescence. The experiments were performed in triplicate

\section{Swertiamarin enhanced ROS production}

The effect of swertiamarin on ROS production was studied using $\mathrm{H}_{2}$ DCFDA staining assay. The results revealed that swertiamarin enhanced intracellular ROS concentration in a dosedependent fashion. The carboxyl- $\mathrm{H}_{2}$ DCFDA fluorescence intensities observed at swertiamarin doses of 10,40 and $80 \mu \mathrm{M}$ were $250,500,1700$ 
and 2200, respectively (Figure 7). Fluorescence intensity was directly proportional to the ROS concentration.

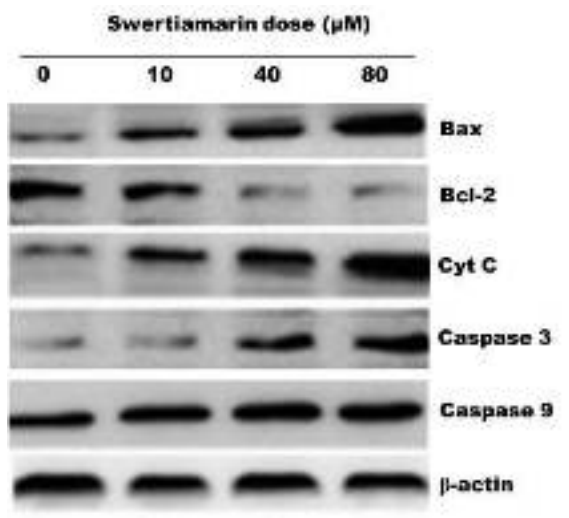

Figure 6: Effect of swertiamarin on expression levels of different apoptosis-related proteins. Data are shown as mean $\pm \mathrm{SD}(\mathrm{n}=3) ; p<0.05$

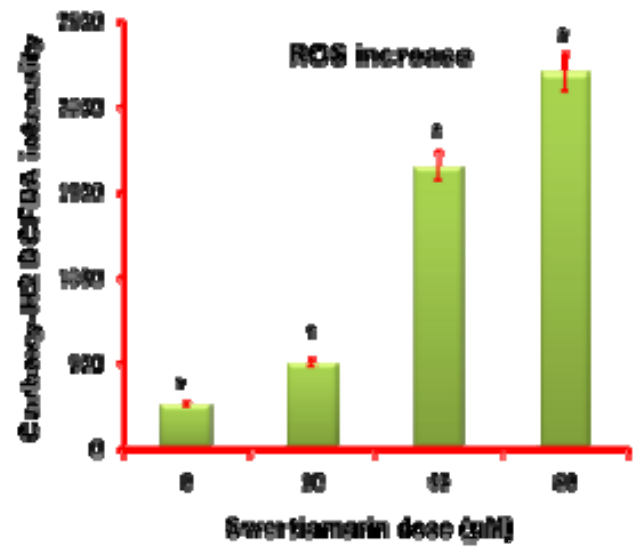

Figure 7: ROS levels after swertiamarin treatment of HeLa cells at indicated doses. $\mathrm{H}_{2}$ DCFDA fluorescence intensity was directly proportional to ROS levels. Data are shown as mean \pm SD $(n=3) ; p<0.05$

\section{Effect of swertiamarin on the migration and invasion of HeLa cells}

Cell migration ability of HeLa CC cells was measured with Transwell migration assay. The results revealed that swertiamarin exerted dosedependent inhibitory effect on the migration of HeLa cells (Figure 8). Furthermore, cell invasion ability of HeLa cells was investigated through Transwell chambers coated with Matrigel, and it was revealed that swertiamarin exposure led to potent and concentration-dependent suppression of invasive ability of HeLa CC cells (Figure 9). Thus, swertiamarin suppressed the migration and invasion of cancerous HeLa cells dosedependently.
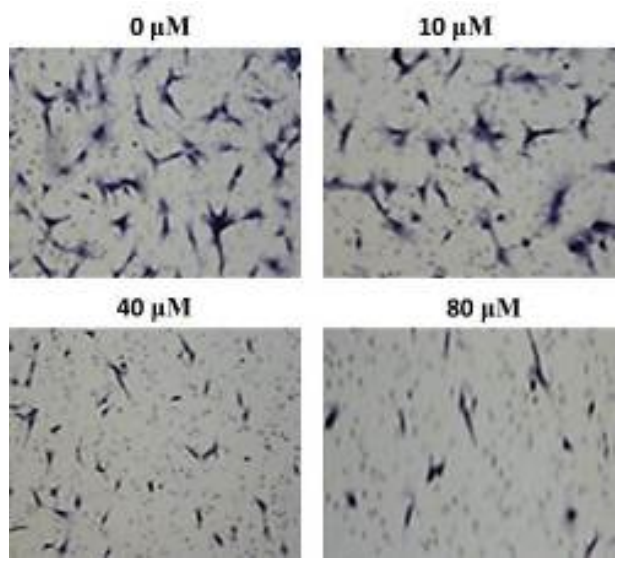

Figure 8: Inhibitory effect of swertiamarin on Hela cells. Experiments were performed in triplicate
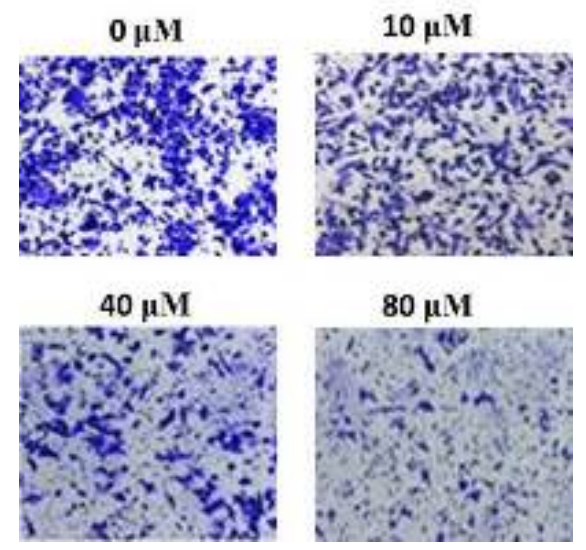

Figure 9: Effect of swertiamarin on invasion of HeLa cells. The experiments were performed in triplicate

\section{Effect of swertiamarin on the MEK/ERK signaling pathway}

Swertiamarin produced anti-proliferative effects on HeLa cells. Therefore, its impact on MEK/ERK signaling pathway was measured using western blotting, since MEK/ERK signaling pathway is important for cell survival and differentiation. The results depicted dosedependent downregulations of the protein expressions of $\mathrm{p}-\mathrm{MEK}$ and $\mathrm{p}-\mathrm{ERK}$, with almost no alterations in MEK and ERK (Figure 10).

\section{DISCUSSION}

Reactive oxygen species (ROS) are extremely reactive by-products of metabolic processes. They exert beneficial and deleterious impacts. Normal physiological functions are regulated by ROS acting as secondary messengers on signaling cascades [21]. 


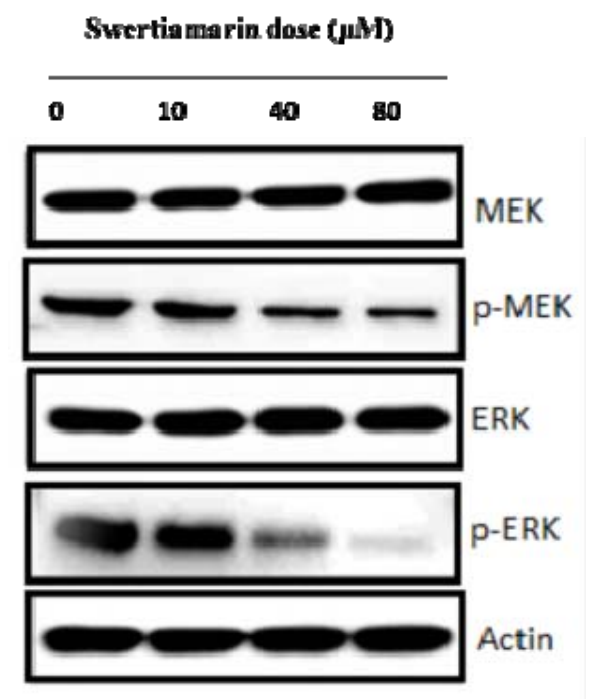

Figure 10: Western blotting assay of expressions of MEK/ERK signaling pathway-associated proteins. Data are shown as mean \pm SD. The experiments were performed in triplicate

Overproduction of ROS is harmful to cells because ROS damage biomolecules i.e. lipids, DNA, proteins and carbohydrates. In the recent past, ROS have been shown to be associated with angiogenesis, tumorigenesis and metastasis. Overproduction and accumulation of ROS in cancer cells lead to cancer cell death.

Studies have also reported that cancer cells contain high ROS levels due to mitochondrial dysfunction and increased metabolic rate. Thus, cancer cells reach the threshold of oxidative stress faster than normal cells. Therefore, bioactive compounds which increase ROS levels are often selected for targeted cancer therapies. In the present study, the anticancer property of swertiamarin was evident in its capacity to mediate ROS production and mitochondriamediated apoptosis, inhibition of cell migration and invasion, and targeting of MEK/ERK signaling pathway. The cytotoxic potency of swertiamarin on HeLa CC cells was monitored with MTT assay which showed that viability of HeLa cells declined significantly with increasing concentrations of swertiamarin and duration of exposure.

The effect of swertiamarin on clonogenic property of HeLa cells was monitored via clonogenic assay which revealed that swertiamarin suppressed the clonogenic potency of the cells. Moreover, results from phase contrast microscopy revealed that swertiamarin caused significant morphological changes on HeLa cells, indicating its apoptotic cell deathinducing potency. Therefore, AO/EB staining was performed to analyse apoptotic cell death in HeLa cells after swertiamarin exposure. The results revealed dose-dependent increases in the number of early, late apoptotic and necrotic cells. Western blotting assay revealed increased protein expression levels of caspase-3, caspase9, Bax and Cyt-c. Thus, the anti-proliferation effect of swertiamarin in HeLa CC cells was mediated through apoptosis. Swertiamarin increased ROS production in a dose dependent manner, and inhibited the migration and invasion potency of HeLa cells. Western blot analysis showed that swertiamarin significantly enhanced the protein expressions of $p-M E K$ and $p$-ERK but had no effect on MEK and ERK even at higher doses of exposure. Thus, swertiamarin blocked the MEK/ERK signaling pathway.

\section{CONCLUSION}

The results obtained in this study indicate that swertiamarin exerts anti-cervical cancer effects via induction of mitochondria-mediated apoptosis, ROS generation, inhibition of cell migration, and invasion and targeting of MEKERK signaling pathway. These findings suggest that swertiamarin may be a potential anti-cervical cancer agent.

\section{DECLARATIONS}

\section{Conflict of interest}

No conflict of interest is associated with this work.

\section{Contribution of authors}

We declare that this work was done by the authors named in this article and all liabilities pertaining to claims relating to the content of this article will be borne by the authors. The whole experiments was performed by Xinxiang Wang under the supervision of Tao Wang.

\section{Open Access}

This is an Open Access article that uses a funding model which does not charge readers or their institutions for access and distributed under the terms of the Creative Commons Attribution License (http://creativecommons.org/licenses/by/ 4.0) and the Budapest Open Access Initiative (http://www.budapestopenaccessinitiative.org/rea d), which permit unrestricted use, distribution, and reproduction in any medium, provided the original work is properly credited.

Trop J Pharm Res, January 2021; 20(1): 80 


\section{REFERENCES}

1. Adlercreutz H, Lignans and human health. Crit Rev Clin Lab Sci 2007; 44: 483-525.

2. Benowitz NL, Clinical pharmacology of caffeine. Annu Rev Med 1990; 41: 277-288.

3. Botta B, Vitali A, Menendez P, Misiti D, Delle Monache $G$, Prenylated flavonoids: pharmacology and biotechnology. Curr Med Chem 2005; 12: 717-739.

4. Wall ME, Wani MC, Cook CE, Palmer KH, McPhail AT, Sim GA. Plant antitumor agents. I. The isolation and structure of camptothecin, a novel alkaloidal leukemia and tumor inhibitor from Camptotheca acuminata. J Am Chem Soc 1966; 88: 3888-3890.

5. Kirtikar KR, Basu $B D$, Indian medicinal plants. 2nd edn, 1935. 3: 1655-1656.

6. Sadique J, Chandra T, Thenmozhi V, Elango $V$, The antiinflammatory activity of Enicostemma littorale and Mollugo cerviana. Biochem Med Metab Biol, 1987; 37: 167-176.

7. Kavimani S, Manisenthlkumar KT, Effect of methanolic extract of Enicostemma littorale on Dalton's ascitic Iymphoma. J Ethnopharmacol 2000; 71: 349-352.

8. Vishwakarma SL, Goyal RK, Hepatoprotective activity of Enicostemma littorale in CCl4-induced liver damage. J Nat Rem 2004; 4: 120-126.

9. Rai J, Thakar KA, Chemical investigation of Enicostemma littorale Blume. Curr Sci, 1966; 35: 148149.

10. Desai PD, Ganguly AK, Govindachari TR, Joshi BS, Kamat VN, Manmade AH, Mohamed PA, Nagle SK, Nayak RH, Saksena AK, Sathe SS, Vishwanathan N, Chemical investigation of some Indian medicinal plants: part II. Indian J Chem 1966; 4: 457-459.
11. Vishwakarma SL, Rajani M, Bagul MS, Goyal RK, A rapid method for the isolation of swertiamarin from Enicostemma littorale. Pharm Biol, 200; 42: 400-403.

12. Tushar P. Patel, Sanket Soni, Pankti Parikh, Jeetendra Gosai, Ragitha Chruvattil, and Sarita Gupta. Swertiamarin: An Active Lead from Enicostemma littorale Regulates Hepatic and Adipose Tissue Gene Expression by Targeting PPAR- $\gamma$ and Improves Insulin Sensitivity in Experimental NIDDM Rat Model. Evid Based Complement Alternat Med 2013; 358673.

13. Hitesh Vaidya, Ramesh K Goyal and Sukhinder Kaur Cheema. Anti-diabetic Activity of Swertiamarin is due to an Active Metabolite, Gentianine, that Upregulates PPAR-g Gene Expression in 3T3-L1 cells. Phytother. Res 2012; 27(4): 624-627.

14. World Cancer Report 2014. World Health Organization. 2014. Chapter 5.12. ISBN 978-9283204299.

15. World Cancer Report 2014. World Health Organization. 2014. pp. Chapter 1.1. ISBN 978-9283204299.

16. Cervical cancer prevention and control saves lives in the Republic of Korea. World Health Organization. 2018.

17. World Health Organization. Fact sheet No. 297. 2014.

18. Canavan TP, Doshi NR. Cervical cancer. Am Fam Physician, 2000. 61 (5): 1369-76.

19. Gadducci A, Barsotti C, Cosio S, Domenici L, Riccardo Genazzani A. Smoking habit, immune suppression, oral contraceptive use, and hormone replacement therapy use and cervical carcinogenesis: A review of the literature. Gynecol Endocrinol 2011. 27 (8): 597-604.

20. Stuart Campbell; Ash Monga. Gynaecology by Ten Teachers (18th edn). Hodder Education. 2006. ISBN 978-0-340-81662-2.

21. L Covarrubias, D Hernandez-Garcia, D Schnabel, E Salas-Vidal, S Castro-Obregon. Function of reactive oxygen species during animal development: passive or active. Developmental Biology, 2008. 320:1-11. 